\subsection{2 \\ ENTEROVIRUSES ISOLATED FROM CHILDREN FROM MIGRANTS' FAMILIES IN THE NORTH-WEST OF RUSSIA}

O.I. Kanaeva, N.I. Romanenkova, N.R. Rozaeva, M.A. Bichurina

St. Petersburg Pasteur Institute, St. Petersburg, Russia

The goal of the study was to compare non-polio enteroviruses isolated from children from migrants' families and from resident children in the North-West of Russia. Isolation of viruses was performed according to the Polio Laboratory Manual, WHO, 2005. The genome region VP1 of certain isolated enterovirus strains was partially sequenced in order to identify virus serotype. WHO Subnational Poliomyelitis Laboratory in St. Petersburg is responsible for 14 administrative territories with the population of more than 20 million people. Annually we investigate nearly three hundred samples from children with acute flaccid paralysis, enterovirus infection, healthy resident children and healthy children under five who arrive in the North-West of Russia from unsafe territories, mostly from Central Asia.

The percentage of enterovirus isolation from healthy children from migrants' families was practically the same as from resident patients with enterovirus infection (about 10\%). Enteroviruses frequently circulating in the North-West of Russia are Echoviruses 6, 9, 11, 13, 30, Coxsackieviruses A4, A6, A10, A16 and Coxsackieviruses B1-6. We found all these enteroviruses in the samples from children from migrants' families. In addition to these enteroviruses we also detected enteroviruses which had not been previously isolated in the North-West of Russia, such as Coxsackieviruses A13, 17, 24, Enteroviruses 75, 99 and 120, Echoviruses 18 and 29. Phylogenic analysis showed that serotypes of enteroviruses which were isolated from migrants' children had a different origin when compared to the viruses of the same serotype regularly detected in the North-West of Russia. Nucleotide sequences of Coxsackieviruses A13 and A17 strains isolated from the children from Tajikistan differed dramatically from sequences presented in GenBank. This may indicate that Coxsackieviruses A strains circulating in the countries of Central Asia have their specific genotype.

Our study proved that the spectra of nonpoliomyelitis enteroviruses isolated from children from migrants' families and from resident children were significantly different. In order to prevent the circulation of imported new enterovirus serotypes and genotypes it is necessary to examine systematically the groups at risk, such as children under 5 who arrive in the North-West of Russia from unsafe territories.

\subsection{3}

doi: 10.15789/2220-7619-2018-4-3.23

\section{DYNAMICS OF MORBIDITY OF THE WEST NILE FEVER IN THE ASTRAKHAN REGION}

S.F. Karpenko ${ }^{1}$, Kh.M. Galimzyanov ${ }^{1}$, B.I. Kantemirova ${ }^{1}$, R.S. Arakelyan ${ }^{1}$, A.R. Kurbangalieva ${ }^{2}$,

G.L. Shendo ${ }^{2}$

${ }^{1}$ Astrakhan State Medical University, Astrakhan, Russia; ${ }^{2}$ Center for Hygiene and Epidemiology in the Astrakhan Region, Astrakhan, Russia

The variety and wide prevalence of arbovirus infections, the possibility of adverse outcomes determine the relevance of their study. In the territory of the Astrakhan re- gion the epidemic focus of West Nile Fever is registered. The purpose of this study was to analyze the dynamics of the morbidity of the West Nile Fever in the Astrakhan region from 2014 to 2017. The analysis of "Data on infectious and parasitic diseases" (Form 1) in the Astrakhan region was carried out.

As our research has shown, West Nile Fever in the Astrakhan region is currently characterized by a low intensity of the epidemic process. 5 people in the Astrakhan region were affected by the West Nile Fever in 2014, the morbidity rate per 100000 of the population was 0.5 . The number of cases increased by 3.0 and 4.8 times respectively in 2015 and 2016. 15 people fell ill with West Nile Fever in 2015, and 24 people - in 2016. The mortality rate per 100000 of the population was equal to 1.5 and 2.4 respectively. It should be noted that the number of people with West Nile Fever in Russian Federation as a whole increased by 1.5 and 4.9 times in 2015 and 2016, compared to 2014. The source of infection in West Nile Fever is mainly wild birds. The increase in the incidence rate in 2015 and 2016 in the Astrakhan region and in Russia may be associated with increased infection of migratory birds during their seasonal migration from the natural foci of West Nile Fever. Only one case of West Nile Fever was registered in the Astrakhan region in 2017, the mortality rate per 100000 of the population decreased in 24 times compared to the previous year and amounted to 0.1 . Children under the age of 14 years were $11.1 \%$ of all the patients with this arbovirus infection from 2014 to 2017.

Thus, the natural focus of the West Nile Fever remains in the Astrakhan region, which activity depends on both the sources of infection and its vectors influenced by the intensity of the epidemic process in endemic foci, seasonal migration of sources of infection and climatic conditions.

\subsection{4}

doi: $10.15789 / 2220-7619-2018-4-3.24$

\section{DETECTION OF PARVOVIRUS INFECTION MARKERS IN RISK GROUPS}

I.V. Khamitova', A.Yu. Antipova ${ }^{1}$, M.Yu. Averyanova ${ }^{2}$,

A.E. Levkovskyi ${ }^{3}$, Yu.V. Ostankova ${ }^{1}$, A.V. Semenov ${ }^{1}$,

A.B. Chukhlovin ${ }^{2}$, I.N. Lavrentieva ${ }^{1}$

${ }^{1}$ St. Petersburg Pasteur Istitute, St. Petersburg, Russia;

${ }^{2}$ R. Gorbacheva Memorial Research Institute of Children Oncology, Hematology and Transplantation, St. Petersburg, Russia; ${ }^{3}$ RUSAL, Fria, Republic of Guine

Parvovirus infection (PVI) caused by Parvovirus B19 (B19V) is transmitted by airborne, parenteral and vertical routes. The virus affects the precursor cells of erythrocytes. PVI can cause serious complications, up to a lethal outcome, in people at risk who are hematological patients, patients with immunosuppression, people requiring blood derived product etc. Despite the high prevalence data on the incidence of $\mathrm{B} 19 \mathrm{~V}$ in people at risk and the clinical manifestations of the disease is not enough.

The aim of the study was to investigate the frequency of occurrence of B19V markers and the effect of PVI on the clinical course and the outcome of initial disease in patients with hematologic profile - malaria patients, children after hematopoietic stem cell transplantation (allo-HSCT).

Plasma/serum samples of malaria patients $(n=316)$ and patients who underwent allo-HSCT $(n=54)$ at the age of 0.6-19 years were examined for the presence of B19V DNA by PCR and IgG-antibodies to B19V by ELISA method. 